\title{
Effects of Standing on Lumbar Spine Alignment and Intervertebral Disc Geometry in Young, Healthy Individuals Determined by Positional Magnetic Resonance Imaging
}

\author{
Christian I. Weber ${ }^{1}$, Ching-Ting Hwang ${ }^{2}$, Linda R. Van Dillen ${ }^{2,3,4}$, and Simon Y. Tang ${ }^{1,4}$ \\ ${ }^{1}$ Biomedical Engineering; Washington University in St. Louis, St. Louis, MO, USA \\ ${ }^{2}$ Movement Science Program; Washington University in St. Louis, St. Louis, MO, USA \\ ${ }^{3}$ Physical Therapy; Washington University in St. Louis, St. Louis, MO, USA \\ ${ }^{4}$ Orthopaedic Surgery Washington University in St. Louis, St. Louis, MO, USA
}

\section{Abstract}

Background: Most diagnostic imaging of the spine is performed in supine, a relatively unloaded position. Although the spine is subjected to functional loading that changes the spinal alignment and intervertebral disc geometry, little data exists on how healthy spines adapt to standing. This study seeks to quantify the changes of the lumbar spine from supine to standing in young, backhealthy individuals using a positional magnetic resonance imaging system.

Methods: This is an observational study that examined the changes in the lumbar spine alignment and intervertebral disc geometry between supine and standing of forty participants (19 males / 21 females) without a history of low back pain. The regional lumbar spinal alignment was measured by the sagittal Cobb angle. Segmental intervertebral disc measurements included the segmental Cobb angle, anterior-to- posterior height ratio, and intervertebral disc width measured at L1/L2 - L5/S1 levels. Intra-class correlation was performed for intra- and inter- observer measurements.

Findings: The intra-observer intra-class correlation consistency model ranged from 0.76-0.98 with inter- observer ranging from $0.68-0.99$. The Cobb angle decreased in standing. The L5/S1 segmental Cobb angle decreased in standing. The L2/L3 and L3/L4 anterior-to-posterior height ratios increased and the L5/S1 anterior-to-posterior height ratio decreased in standing. No difference in intervertebral disc widths were observed from supine to standing.

Interpretations: We established normative data for a back-healthy population, using a positional magnetic resonance imaging system, that could inform future investigations that examine the standing- induced adaptations of the lumbar spine in individuals with spinal or intervertebral disc pathologies.

Corresponding author: Simon Y. Tang, Ph.D. 660 South Euclid Ave, Box 8233, St. Louis, MO 63110, USA, simon.tang@wustl.edu.

Publisher's Disclaimer: This is a PDF file of an unedited manuscript that has been accepted for publication. As a service to our customers we are providing this early version of the manuscript. The manuscript will undergo copyediting, typesetting, and review of the resulting proof before it is published in its final citable form. Please note that during the production process errors may be discovered which could affect the content, and all legal disclaimers that apply to the journal pertain. 


\section{Keywords}

Intervertebral disc; spine; positional MRI; standing; back-healthy

\section{Introduction}

The degeneration of the intervertebral disc (IVD) is a significant contributor to low back pain (LBP) (de Schepper et al., 2010). Despite the association between IVD degeneration and LBP, the correspondence between the clinical presentation of LBP and IVD imaging findings is quite poor (Rea et al., 2012). One possibility for this lack of specificity may be that most diagnostic imaging of the spine is performed in supine, a minimally loaded position (Baker, 2014; Wilke et al., 2006). An individual in standing generates lumbar intradiscal pressures approximately five times greater than in supine (NACHEMSON, 1981) and complex multiaxial forces (Naserkhaki et al., 2016) that result in different tensile and shear deformations across the IVD (Aiyangar et al., 2013; Wang et al., 2009). Compressive loading similar to that experienced during standing is critical for maintaining spinal curvature (Wessberg et al., 2006). Finite element methods have been used to explore the impact of the loads in standing on the lumbar spine (Dreischarf et al., 2016; Liu et al., 2018; Rohlmann et al., 2009), but the generalizability of these findings remain unclear given the number of assumptions that are required to accurately model a complex in vivo system such as the spine. Direct observation of the spine in standing in vivo is thus the most functionally and clinically relevant approach to determine adaptations at the whole joint level.

Magnetic Resonance Imaging (MRI) is a commonly used radiographic technique for the imaging of the spine and for the visualization of hydrated tissue structures such as the IVDs. Positional MRI (pMRI) with an open magnet configuration enables the imaging of human participants in different positions (Alyas et al., 2008; Jinkins et al., 2005; J.R. Jinkins et al., 2002); thus, the pMRI provides the opportunity to observe the spinal structures in loaded positions (Berry et al., 2017; Jinkins et al., 2005; Rodríguez- Soto et al., 2017, 2013). Examining the lumbar spine in an upright position using a pMRI improved the specificity in discriminating acute and chronic LBP populations (Tarantino et al., 2013) and increased the reliability of observing stenosis and IVD degeneration (Hansen et al., 2018). Prior studies have utilized pMRI to examine spinal adaptations between sitting to standing in chronic LBP patients (Karadimas et al., 2006), supine to standing in athletes (Mauch et al., 2010), patients with lumbar spinal stenosis (Madsen et al., 2008), and changes of the dural sac in the lumbar spine due to posture (Hirasawa et al., 2007). Yet examining how a non-diseased population adapts to an increase of spinal loads is critical for understanding the initiating features of LBP. Thus, data from a young, healthy population without a history of LBP ('back-healthy') will help define the norms of loading-induced spinal adaptations and distinguish the deviations from the norm. Moreover, exploring the lumbar spine at the regional and segmental scales could reveal local anomalies that contribute to the initiation of LBP. Backhealthy individuals are also susceptible to developing LBP symptoms in prolonged standing with a high rate of developing chronic LBP later in their life (Gallagher and Callaghan, 2015; Gregory and Callaghan, 2008; Marshall et al., 2011; Nelson-Wong et al., 2008; Nelson-Wong and Callaghan, 2014; Sorensen et al., 2015). Therefore, examining the 
standing-induced adaptations in the back-healthy population may provide predictive information prior to development of spinal pathologies.

The pMRI can be leveraged to observe adaptations across positions of the lumbar spine using regional alignment measurements (sagittal Cobb angle) and measurements at each level of the lumbar spine such as segmental Cobb angle, anterior-to-posterior height (A/P) ratio, and IVD width. Therefore, the objectives of this study are to 1) investigate the effects of standing on the spinal alignment and individual lumbar IVDs of young, back-healthy human participants ranging from 18 to 30 years of age using pMRI, and 2) determine whether there are sex-specific differences in these measurements due to the variations in spinal alignment between males and females (Hay et al., 2015; Youdas et al., 1996). We hypothesized that 1) the sagittal Cobb angle would be significantly different between supine and standing, 2) individual IVDs across the lumbar spine would adapt differentially to standing, and 3) the change in segmental Cobb angle, A/P ratio, and IVD width at each lumbar level from supine to standing would be different between males and females.

\section{Methods}

\subsection{Participants}

The study included forty participants (19 male / 21 female) between 18-30 years of age and body-mass index $(\mathrm{BMI})<30 \mathrm{~kg} / \mathrm{m}^{2}$ (Table 1). Participants were recruited through posted flyers and distributed emails to the community and local universities in the St. Louis metropolitan area. Participants were excluded during screening if they reported any history of LBP. LBP was defined as pain in the lumbar region greater than 2 on a $0-10$ verbal numeric rating scale that lasted at least 24 hours that resulted in one or more of the following: (1) some type of medical intervention (e.g., physician, physical therapist, chiropractor); (2) three or more consecutive days of missed work or school; (3) three of more consecutive days of altered activities of daily living. Exclusion criteria also included a prior diagnosis of diabetes, anxiety, depression, employment in a job that involved standing for greater than 4 hours per day or standing in one place for more than 1 hour per day during the last 12 months, consumption of caffeinated drinks $>25$ per week, consumption of alcoholic drinks $>10$ per week, or smoking cigarettes $>15$ per day. None of the female participants identified with being currently or recently pregnant. All participants read and signed an informed consent form in accordance with the Human Research Protection Office at Washington University School of Medicine. The participants were instructed to avoid nonhabitual strenuous physical activity (e.g., running, weight lifting) for 24 hours prior to imaging with no rescheduling required. All imaging was performed in the afternoon after 12PM to minimize diurnal variations (Ludescher et al., 2008).

\subsection{Data collection}

Images of the lumbar spine (L1-S1) were obtained using the 0.6T Open UPRIGHT® MRI (Fonar, New York, NY) system. A 3-plane localizer was used to acquire sagittal T2 weighted images (repetition time $=610 \mathrm{~ms}$, echo time $=17 \mathrm{~ms}$, field of view $=24 \mathrm{~cm}$, acquisition matrix $=210 \times 210$, slice thickness $=3 \mathrm{~mm}$, no gap, scan duration $=2 \mathrm{~min}$ ) (Rodríguez-Soto et al., 2013). This sequence was optimized for reducing scan time and motion artifacts [12- 
13]. A wood plank was placed adjacent to the quad-planar coil on the MRI table to provide a continuous flat surface for the lumbar spine (Fig. 1). The participant entered the scanner facing forward with his/her back against the table. A pillow was placed behind the head to provide support to the neck. The table was adjusted to a horizontal position of 180 degrees. The participant was positioned in supine for 10 minutes prior to the first scan. The MRI table then was moved to a vertical position with a 84 degree table tilt where participants were then standing. The table tilt of 84 degrees helped to stabilize the participant and prevent motion artifacts during the imaging in standing (Fig. 1). The pillow was removed, and a VersaRest ${ }^{\mathrm{TM}}$ device, an arm support, was placed in the scanner underneath the wrists 5 $\mathrm{cm}$ below the lateral epicondyle of the elbow (Fig. 1). Participants were told to stand normally without leaning on the sides of the magnet, back of the scanner or on the VersaRest ${ }^{\mathrm{TM}}$ during the scan in standing. A board-certified MRI technician conducted all imaging. After imaging, all images were exported as DICOM files to be analyzed on Miele (OsiriX)-LXIV (open source) (Rosset et al., 2004).

\subsection{Regional Lumbar Spine Alignment}

The four-line Cobb method, denoted here as the Cobb angle, was used to quantify regional lumbar spine alignment (Harrison et al., 2001). The Cobb angle was measured between the inferior T12 and superior S1 endplates (Fig. 2A). This measurement was obtained in supine and standing for each participant using the mid-sagittal slice of the MR image.

\subsection{Segmental IVD Measurements}

The measures for each of the five lumbar IVDs, L1/L2 through L5/S1, were obtained in supine and standing for each participant using the mid-sagittal slice of the MR image. The measures included the segmental Cobb angle, the anterior-to-posterior height $(\mathrm{A} / \mathrm{P})$ ratio, and the IVD width (Fig. 2B, 2C and 2D). The segmental Cobb angle was calculated as the angle created by the line segment containing the anterior and posterior edges of the superior endplate with the line segment containing anterior and posterior side of the inferior endplate (Fig. 2B). The heights were calculated as the distances on the superior endplate to inferior endplate distance at the anterior and posterior sides for each IVD level. The IVD width was defined as the maximum distance between the anterior and posterior bulge points of an IVD in a sagittal view (Fig. 2C). The A/P ratio was calculated by dividing the anterior height by the posterior height (Fig. 2D).

\subsection{Reproducibility, error and uncertainty}

The intra-observer (C.W./researcher) and inter-observer (researchers) reproducibility were indexed with the Intraclass Correlation Coefficient (ICC) using data from participants that exhibited the greatest range of values for regional and all lumbar segments (McGraw and Wong, 1996). A written rubric based on anatomical landmarks (segmental Cobb angles, IVD widths, the anterior-, and posterior- heights) was used for all segmental measurements. The intra-observer analysis was performed using measured variables obtained on three distinct nonconsecutive days. Additional observers performed the inter- observer analysis based on the written rubric for regional and segmental measurements with three sets of measurements on non-consecutive days. 
We quantified the error associated with image resolution and its impact on the uncertainty of IVD measurements. The resolution-based error of the MRI images was determined by moving the coordinates of each anatomical landmark in every possible direction and calculating the effect on the subsequent IVD measurements. The maximum uncertainty from resolution on the measurements using this combinatorial approach were $5.2 \%, 5.4 \%$, and $1.3 \%$ for segmental Cobb angle, A/P ratio, and IVD width, respectively.

\subsection{Statistical Analyses}

A three-way, repeated measures analysis of variance (ANOVA) was used to test for the factors of position, sex, and lumbar level for each of the three segmental measurements (segmental Cobb angles, A/P ratios, and IVD widths). Cobb angle was analyzed using a two-way repeated measures ANOVA to test for the factors of position and sex. Interaction terms are denoted by an asterisk (*) in Table 2 and in the results. Post-hoc comparisons were done using the Fisher's least significant difference (LSD) test. Differences are significant when the associated $\mathrm{p}$-value is $\leq 0.05$. Post-hoc power analysis was performed on significant results using G*Power (Faul et al., 2007).

\section{Results}

All enrolled participants $(n=40)$ completed the supine and standing imaging sessions and were included in all analyses.

\subsection{Reliability of Measurements}

The ICC values (with the 95\% confidence interval in parentheses) for the segmental Cobb angle, IVD width, and the anterior and posterior height measurements were $0.90(0.79-$ $0.96), 0.98$ (0.95-0.99), 0.89 (0.78-0.95), and $0.76(0.58-0.89)$ respectively (Fig. 3). The inter-observer ICC agreement for segmental Cobb angle, IVD width, and the anterior and posterior height measurements were 0.89 (0.79-0.95), $0.99(0.98-1), 0.84(0.7-0.93)$, and $0.68(0.46-0.85)$, respectively. The intra-observer ICC for Cobb angle was $0.97(0.90-$ $0.99)$, and the inter-observer ICC was $0.87(0.61-0.97)$.

\subsection{Regional Lumbar Spine Alignment}

Position was a significant factor. The Cobb angle decreased in standing compared to supine for all participants (Fig. 4, Table 3; $P<0.05$ ).

\subsection{Segmental IVD Measurements}

3.3.1 Segmental Cobb Angle-Sex, level, and level*position all were significant factors (Table 2). Post hoc analyses revealed that compared to supine the L5/S1 segmental Cobb angle decreased in standing (Fig. 5, Table 3; $P<0.05$ ).

3.3.2 A/P ratio-Sex, level, position, and level*position were significant factors (Table 2). Compared to supine the $\mathrm{L} 2 / \mathrm{L} 3$ and $\mathrm{L} 3 / \mathrm{L} 4 \mathrm{~A} / \mathrm{P}$ ratio increased in standing $(P<0.05)$, and the L5/S1 A/P ratio decreased in standing (Fig. 6, Table 3; $P<0.05$ ). 
3.3.3 IVD Width-Sex, level, position, and level*position were significant factors (Table 2). There were no significant differences in IVD width due to positions at lumbar levels L1/L2 to L4/L5 for all participants (Table 3). Although the L5/S1 IVD width in standing was statistically greater than in supine $(P<0.05)$, the margin of the difference $(0.48 \%)$ was below the detection threshold per our error analyses $(1.3 \%)$, and thus we did not consider this difference to be meaningful.

\section{Discussion}

To our knowledge this is the first study to examine the segmental Cobb angle, A/P ratio and IVD width from supine to standing in back-healthy males and females. Consequently, the objectives of this study was to investigate the effects of standing on the segmental measurements and examine the sex differences in these adaptations. Our results indicate that the sagittal Cobb angle was significantly decreased in standing compared to supine. Individual IVDs across the lumbar spine exhibited adaptations that were level-dependent. Surprisingly, we did not observe sex-dependent changes.

While other studies examined angular changes that included the vertebrae and the adjacent IVD (Lee et al., 2014; Wood et al., 1996), in this study we measured the changes between the inferior and superior vertebral endplates of individual discs enabling observation of IVDspecific adaptations at every lumbar level (Acosta et al., 2011; Hsieh et al., 2007). pMRI studies on Marine populations have shown that individual IVDs adapt to external loads placed on the spine in standing (Berry et al., 2017; Rodríguez-Soto et al., 2013). The changes in segmental IVD measurements from supine to standing in our back-healthy group show that adaptations due to increased loading occur at multiple levels of the lumbar spine, similar to the externally loaded spine in the supine position (Kimura et al., 2001). The ability of these lumbar levels to adapt may be important for the spine to distribute loads, especially when the diminished ability of individual lumbar levels to adapt to loading are concomitant with disease and degeneration (Berry et al., 2017; Kulig et al., 2007). Given that the pMRI in standing improves the detection of IVD bulging (Zou et al., 2008), the lack of bulging in these participants in standing confirms that these IVDs are indeed relatively healthy (Adams et al., 2000; Stefanakis et al., 2014).

The reduced lordosis of our participants in standing is consistent with the observations of another pMRI study (Madsen et al., 2008). Other studies using a pMRI have shown that lordosis is not different between supine and standing with a cushion underneath their knees (Hirasawa et al., 2007). These results are in contrast with studies done using plain film radiography which observed more lordosis in standing (Chevillotte et al., 2018; Lee et al., 2014; Wood et al., 1996). The discrepancy is likely due to a pelvic tilt that has been shown to influence lumbar lordosis in standing (Levine and Whittle, 1996), since the pelvis is strongly correlated to lumbar lordosis in supine (Chevillotte et al., 2018). In this study, we did not directly control for pelvic tilt by placing a pillow underneath the legs in supine. Despite this, the measured Cobb angles in standing are consistent with a prior report that also included young, asymptomatic individuals (Yukawa et al., 2018). Further, it has been shown that externally applied loads during standing can alter the lumbar spine to be in less lordosis in pMRI (Berry et al., 2017; Rodríguez-Soto et al., 2017, 2013). 
The data shows that the lordosis is not different between sexes as previously reported (Hay et al., 2015; Youdas et al., 1996). Additionally, we observed that males and females comparably change from supine to standing. Wood et al. included males and females in their study but did not specifically report on sex differences in position (Wood et al., 1996). Although we observed sex as a significant factor in the segmental measurements, there were no interactions between sex and position, suggesting though both sexes adapt to standing, they do not adapt differently. In this study, the decline of the Cobb angle of the spine in standing in a young, back-healthy group with no observed translational lumbosacral anatomies or spondylolysis/spondylolisthesis is coupled with measurable changes in the IVD across the different lumbar levels (Fig. 5, 6).

Characterizing the IVD using measurements that are based on independent landmarks is important for capturing the nuanced changes in the structure of the IVD (Hansen et al., 2018; Hirasawa et al., 2007; Tarantino et al., 2013). Using the pMRI system, we demonstrate that it is possible to determine and reliably characterize the regional lumbar alignment and individual lumbar IVDs using the segmental measurements of segmental Cobb angle, A/P ratio, and IVD width. Moreover, the images obtained in standing can yield more physiologically relevant information than a slightly higher resolution scan taken in supine due to the differences in loading between supine and standing. Although some of the measurements made here could also be obtained from plain film radiographs (Lee et al., 2014; Lord et al., 1997), the pMRI enables the possibility of assessing any potential involvement of IVD-specific pathologies (such as herniations) in the spinal structural measurements. The nature of MRI also leverages the increased signal of hydrated microstructures in the IVD, and the 3D reconstruction capabilities of the volumetric image stack could be utilized further for additional spatially-unbiased analyses.

There are several limitations to our study. First, although the field strength of this pMRI system $(0.6 \mathrm{~T})$ is lower than typical clinical systems $(1.5 \mathrm{~T})$, the higher efficiency of the field algorithm reconstructs and generates images at comparable resolutions to $1.5 \mathrm{~T}$ systems (Hansson et al., 1989). The ICC and uncertainty analyses confirm the reliability of the measurements using the pMRI system. The inter-observer reliability ranged from 0.68 to 0.99 and is consistent with a MRI study that reported an inter-observer ICC range of $0.73-$ 0.95 (Guen et al., 2011). Analyses of error and uncertainty in the images reveal that the resolution can affect the measurements by $1.3 \%-5.4 \%$, and thus only differences that were greater than the uncertainty range were considered to be meaningful. Second, the pelvic tilt, which was not directly controlled for and this may have contributed to the participants' variations in the supine spinal alignment. Third, although we only enrolled 40 participants in this study, the power for our discriminating measurements ranged from 0.57-0.99, indicating that the sample size is adequate for most measures. Only the L3/L4 A/P ratio was powered below 0.80 at 0.57 .

\section{Conclusions}

This study is the first to examine the differences in regional and segmental measurements between supine and standing positions in young, back-healthy participants using a pMRI system. Further, we have developed and validated a method using the pMRI system to 
evaluate changes in spinal measurements. Our key finding here is that the individual IVD levels within a healthy spine adapt to loaded positions non-uniformly. These findings highlight the importance of acquiring images in standing as they are different than the traditional MRI images in supine. Observing IVDs in standing and comparing symptomatic and asymptomatic individuals can be more informative of IVD function and help elucidate the discordance between imaging findings and LBP.

\section{ACKNOWLEDGEMENTS}

These studies were supported by the National Institutes of Health (P30 AR57325 - WUSTL Musculoskeletal Research Center), K01AR069116, R21AR069804, and 5T32EB018266. The authors gratefully acknowledge Dave Magner, Travis Gould, Nicholas Woerther and the staff at The Open Upright MRI of Missouri for providing technical assistance with the imaging. The authors thank Kaitlyn Broz for her critical readings of the manuscript.

\section{References}

Acosta FL, Liu J, Slimack N, Moller D, Fessler R, Koski T, 2011 Changes in coronal and sagittal plane alignment following minimally invasive direct lateral interbody fusion for the treatment of degenerative lumbar disease in adults: a radiographic study. J. Neurosurg. Spine 15, 92-96. 10.3171/2011.3.SPINE10425 [PubMed: 21476802]

Adams MA, Freeman BJC, Morrison HP, Nelson IW, Dolan P, 2000 Mechanical Initiation of Intervertebral Disc Degeneration. Spine 25.

Aiyangar AK, Zheng L, Tashman S, Anderst WJ, Zhang X, 2013 Capturing Three-Dimensional In Vivo Lumbar Intervertebral Joint Kinematics Using Dynamic Stereo-X-Ray Imaging. J. Biomech. Eng 136, 011004-011004-9. 10.1115/1.4025793

Alyas F, Connell D, Saifuddin A, 2008 Upright positional MRI of the lumbar spine. Clin. Radiol 63, 1035-1048. 10.1016/j.crad.2007.11.022 [PubMed: 18718234]

Baker ADL, 2014 Abnormal Magnetic-Resonance Scans of the Lumbar Spine in Asymptomatic Subjects. A Prospective Investigation, in: Banaszkiewicz PA, Kader DF (Eds.), Classic Papers in Orthopaedics. Springer London, London, pp. 245-247. 10.1007/978-1-4471-5451-8_60

Berry DB, Rodríguez-Soto AE, Su J, Gombatto SP, Shahidi B, Palombo L, Chung C, Jensen A, Kelly KR, Ward SR, 2017 Lumbar spine postures in marines during simulated operational positions. J. Orthop. Res n/a-n/a. 10.1002/jor.23510

Chevillotte T, Coudert P, Cawley D, Bouloussa H, Mazas S, Boissière L, Gille O, 2018 Influence of posture on relationships between pelvic parameters and lumbar lordosis: Comparison of the standing, seated, and supine positions. A preliminary study. Orthop. Traumatol. Surg. Res 104, 565568. 10.1016/j.otsr.2018.06.005 [PubMed: 30009961]

de Schepper EIT, Damen J, van Meurs JBJ, Ginai AZ, Popham M, Hofman A, Koes BW, BiermaZeinstra SM, 2010 The Association Between Lumbar Disc Degeneration and Low Back Pain: The Influence of Age, Gender, and Individual Radiographic Features. Spine 35, 531-536. 10.1097/BRS. 0b013e3181aa5b33 [PubMed: 20147869]

Dreischarf M, Shirazi-Adl A, Arjmand N, Rohlmann A, Schmidt H, 2016 Estimation of loads on human lumbar spine: A review of in vivo and computational model studies. J. Biomech., SI: Spine Loading and Deformation 49, 833-845. 10.1016/j.jbiomech.2015.12.038

Faul F, Erdfelder E, Lang A-G, Buchner A, 2007 G*Power 3: A flexible statistical power analysis program for the social, behavioral, and biomedical sciences. Behav. Res. Methods 39, 175-191. 10.3758/BF03193146 [PubMed: 17695343]

Gallagher KM, Callaghan JP, 2015 Early static standing is associated with prolonged standing induced low back pain. Hum. Mov. Sci 44, 111-121. 10.1016/j.humov.2015.08.019 [PubMed: 26340276]

Gregory DE, Callaghan JP, 2008 Prolonged standing as a precursor for the development of low back discomfort: An investigation of possible mechanisms. Gait Posture 28, 86-92. 10.1016/j.gaitpost. 2007.10.005 [PubMed: 18053722] 
Guen YL, Joon WL, Hee SC, Kyoung-Jin O, Heung SK, 2011 A new grading system of lumbar central canal stenosis on MRI: an easy and reliable method. Skeletal Radiol. 40, 1033-1039. 10.1007/ s00256-011-1102-x [PubMed: 21286714]

Hansen BB, Hansen P, Christensen AF, Trampedach C, Rasti Z, Bliddal H, Boesen M, 2018 Reliability of standing weight-bearing $(0.25 \mathrm{~T}) \mathrm{MR}$ imaging findings and positional changes in the lumbar spine. Skeletal Radiol. 47, 25-35. 10.1007/s00256-017-2746-y [PubMed: 28812185]

Hansson L, Westesson P, Katzberg R, Tallents R, Kurita K, Holtas S, Svensson S, Eriksson L, Johansen C, 1989 MR imaging of the temporomandibular joint: comparison of images of autopsy specimens made at $0.3 \mathrm{~T}$ and $1.5 \mathrm{~T}$ with anatomic cryosections. Am. J. Roentgenol 152, $1241-$ 1244. 10.2214/ajr.152.6.1241 [PubMed: 2718861]

Harrison DE, Harrison DD, Cailliet R, Janik TJ, Holland B, 2001 Radiographic analysis of lumbar lordosis: centroid, Cobb, TRALL, and Harrison posterior tangent methods. Spine 26, E235-242. [PubMed: 11389407]

Hay O, Dar G, Abbas J, Stein D, May H, Masharawi Y, Peled N, Hershkovitz I, 2015 The Lumbar Lordosis in Males and Females, Revisited. PLOS ONE 10, e0133685 10.1371/journal.pone. 0133685 [PubMed: 26301782]

Hirasawa Y, Bashir WA, Smith FW, Magnusson ML, Pope MH, Takahashi K, 2007 Postural Changes of the Dural Sac in the Lumbar Spines of Asymptomatic Individuals Using Positional Stand-Up Magnetic Resonance Imaging: Spine 32, E136-E140. 10.1097/01.brs.0000255202.94153.ca [PubMed: 17304123]

Hsieh PC, Koski TR, O’Shaughnessy BA, Sugrue P, Salehi S, Ondra S, Liu JC, 2007 Anterior lumbar interbody fusion in comparison with transforaminal lumbar interbody fusion: implications for the restoration of foraminal height, local disc angle, lumbar lordosis, and sagittal balance. J. Neurosurg. Spine 7, 379-386. 10.3171/SPI-07/10/379 [PubMed: 17933310]

Jinkins JR, Dworkin JS, Damadian RV, 2005 Upright, weight-bearing, dynamic-kinetic MRI of the spine: initial results. Eur. Radiol 15, 1815-1825. 10.1007/s00330-005-2666-4 [PubMed: 15906040]

Jinkins JR, Dworkin JS, Green CA, Greenhalgh JF, Gianni M, Gelbien M, Wolf RB, Damadian J, Damadian RV, 2002 Upright, Weight-Bearing, Dynamic-Kinetic MRI of the Spine pMRI/kMRI. Riv. Neuroradiol 15, 333-357. 10.1177/197140090201500404

Karadimas EJ, Siddiqui M, Smith FW, Wardlaw D, 2006 Positional MRI Changes in Supine Versus Sitting Postures in Patients With Degenerative Lumbar Spine. Clin. Spine Surg 19, 495 10.1097/01.bsd.0000211213.98070.c2

Kimura S, Steinbach GC, Watenpaugh DE, Hargens AR, 2001 Lumbar Spine Disc Height and Curvature Responses to an Axial Load Generated by a Compression Device Compatible with Magnetic Resonance Imaging. [Miscellaneous Article]. Spine 26, 2596-2600. [PubMed: $11725241]$

Kulig K, Powers CM, Landel RF, Chen H, Fredericson M, Guillet M, Butts K, 2007 Segmental lumbar mobility in individuals with low back pain: in vivo assessment during manual and self-imposed motion using dynamic MRI. BMC Musculoskelet. Disord 8, 8 10.1186/1471-2474-8-8 [PubMed: 17261197]

Lee ES, Ko CW, Suh SW, Kumar S, Kang IK, Yang JH, 2014 The effect of age on sagittal plane profile of the lumbar spine according to standing, supine, and various sitting positions. J. Orthop. Surg 9, 11 10.1186/1749-799X-9-11

Levine D, Whittle MW, 1996 The Effects of Pelvic Movement on Lumbar Lordosis in the Standing Position. J. Orthop. Sports Phys. Ther. 24, 130-135. 10.2519/jospt.1996.24.3.130

Liu T, Khalaf K, Naserkhaki S, El-Rich M, 2018 Load-sharing in the lumbosacral spine in neutral standing \& flexed postures - A combined finite element and inverse static study. J. Biomech, 2nd International Workshop on Spine Loading and Deformation 70, 43-50. 10.1016/j.jbiomech. 2017.10.033 [PubMed: 29153706]

Lord MJ, Small JM, Dinsay JM, Watkins RG, 1997 Lumbar lordosis: effects of sitting and standing. Spine 22, 2571-2574. [PubMed: 9383867] 
Ludescher B, Effelsberg J, Martirosian P, Steidle G, Markert B, Claussen C, Schick F, 2008 T2- and diffusion-maps reveal diurnal changes of intervertebral disc composition: An in vivo MRI study at 1.5 Tesla. J. Magn. Reson. Imaging 28, 252-257. 10.1002/jmri.21390 [PubMed: 18581387]

Madsen R, Jensen TS, Pope M, Sørensen JS, Bendix T, 2008 The Effect of Body Position and Axial Load on Spinal Canal Morphology: An MRI Study of Central Spinal Stenosis. Spine 33, 61-67. 10.1097/BRS.0b013e31815e395f [PubMed: 18165750]

Marshall PWM, Patel H, Callaghan JP, 2011 Gluteus medius strength, endurance, and co-activation in the development of low back pain during prolonged standing. Hum. Mov. Sci 30, 63-73. 10.1016/ j.humov.2010.08.017 [PubMed: 21227522]

Mauch F, Jung C, Huth J, Bauer G, 2010 Changes in the Lumbar Spine of Athletes From Supine to the True-Standing Position in Magnetic Resonance Imaging: Spine 35, 1002-1007. 10.1097/BRS. 0b013e3181bdb2d3 [PubMed: 20407340]

McGraw KO, Wong SP, 1996 Forming inferences about some intraclass correlation coefficients. Psychol. Methods 1, 30-46. 10.1037/1082-989X.1.1.30

NACHEMSON AL, 1981 Disc Pressure Measurements. Spine 6.

Naserkhaki S, Jaremko JL, Adeeb S, El-Rich M, 2016 On the load-sharing along the ligamentous lumbosacral spine in flexed and extended postures: Finite element study. J. Biomech., SI: Spine Loading and Deformation 49, 974-982. 10.1016/j.jbiomech.2015.09.050

Nelson-Wong E, Callaghan JP, 2014 Transient Low Back Pain Development During Standing Predicts Future Clinical Low Back Pain in Previously Asymptomatic Individuals. Spine 39, E379 10.1097/ BRS.0000000000000191 [PubMed: 24384659]

Nelson-Wong E, Gregory DE, Winter DA, Callaghan JP, 2008 Gluteus medius muscle activation patterns as a predictor of low back pain during standing. Clin. Biomech 23, 545-553. 10.1016/ j.clinbiomech.2008.01.002

Rea W, Kapur S, Mutagi H, 2012 Intervertebral disc as a source of pain. Contin. Educ. Anaesth. Crit. Care Pain 12, 279-282. 10.1093/bjaceaccp/mks028

Rodríguez-Soto AE, Berry DB, Palombo L, Valaik E, Kelly KR, Ward SR, 2017 Effect of Load Magnitude and Distribution on Lumbar Spine Posture in Active-duty Marines: SPINE 42, 345351. 10.1097/BRS.0000000000001742 [PubMed: 27310023]

Rodríguez-Soto AE, Jaworski R, Jensen A, Niederberger B, Hargens AR, Frank LR, Kelly KR, Ward SR, 2013 Effect of Load Carriage on Lumbar Spine Kinematics: Spine 38, E783-E791. 10.1097/ BRS.0b013e3182913e9f [PubMed: 23524870]

Rohlmann A, Zander T, Rao M, Bergmann G, 2009 Applying a follower load delivers realistic results for simulating standing. J. Biomech 42, 1520-1526. 10.1016/j.jbiomech.2009.03.048 [PubMed: 19433325]

Rosset A, Spadola L, Ratib O, 2004 OsiriX: An Open-Source Software for Navigating in Multidimensional DICOM Images. J. Digit. Imaging 17, 205-216. 10.1007/s10278-004-1014-6 [PubMed: 15534753]

Sorensen CJ, Norton BJ, Callaghan JP, Hwang C-T, Van Dillen LR, 2015 Is lumbar lordosis related to low back pain development during prolonged standing? Man. Ther 20, 553-557. 10.1016/j.math. 2015.01.001 [PubMed: 25637464]

Stefanakis M, Luo J, Pollintine P, Dolan P, Adams MA, 2014 ISSLS Prize Winner: Mechanical Influences in Progressive Intervertebral Disc Degeneration. Spine 39, 1365-1372. 10.1097/BRS. 0000000000000389 [PubMed: 24831499]

Tarantino U, Fanucci E, Iundusi R, Celi M, Altobelli S, Gasbarra E, Simonetti G, Manenti G, 2013 Lumbar spine MRI in upright position for diagnosing acute and chronic low back pain: statistical analysis of morphological changes. J. Orthop. Traumatol 14, 15-22. 10.1007/s10195-012-0213-z [PubMed: 22983676]

Wang S, Xia Q, Passias P, Wood K, Li G, 2009 Measurement of geometric deformation of lumbar intervertebral discs under in-vivo weightbearing condition. J. Biomech 42, 705-711. 10.1016/ j.jbiomech.2009.01.004 [PubMed: 19268946]

Wessberg P, Danielson BI, Willén J, 2006 Comparison of Cobb Angles in Idiopathic Scoliosis on Standing Radiographs and Supine Axially Loaded MRI: Spine 31, 3039-3044. 10.1097/01.brs. 0000249513.91050.80 [PubMed: 17173001] 
Wilke H-J, Rohlmann F, Neidlinger-Wilke C, Werner K, Claes L, Kettler A, 2006 Validity and interobserver agreement of a new radiographic grading system for intervertebral disc degeneration: Part I. Lumbar spine. Eur. Spine J 15, 720-730. 10.1007/s00586-005-1029-9 [PubMed: 16328226]

Wood KB, Kos P, Schendel M, Persson K, 1996 Effect of Patient Position on the Sagittal-Plane Profile of the Thoracolumbar Spine. J. Spinal Disord 9, 165-169. [PubMed: 8793786]

Youdas JW, Garrett TR, Harmsen S, Suman VJ, Carey JR, 1996 Lumbar lordosis and pelvic inclination of asymptomatic adults. Phys. Ther 76, 1066-1081. [PubMed: 8863760]

Yukawa Y, Kato F, Suda K, Yamagata M, Ueta T, Yoshida M, 2018 Normative data for parameters of sagittal spinal alignment in healthy subjects: an analysis of gender specific differences and changes with aging in 626 asymptomatic individuals. Eur. Spine J 27, 426-432. 10.1007/ s00586-016-4807-7

Zou J, Yang H, Miyazaki M, Wei F, Hong SW, Yoon SH, Morishita Y, Wang JC, 2008 Missed Lumbar Disc Herniations Diagnosed With Kinetic Magnetic Resonance Imaging. Spine 33, E140 10.1097/ BRS.0b013e3181657f7e [PubMed: 18317181] 


\section{Highlights}

- Intra-observer intra-class correlation ranged 0.76-0.98; inter-observer 0.680.99

- Cobb angle decreased in young, healthy humans in standing in positional MRI

- Healthy individual intervertebral disc levels adapt to loaded positions nonuniformly 

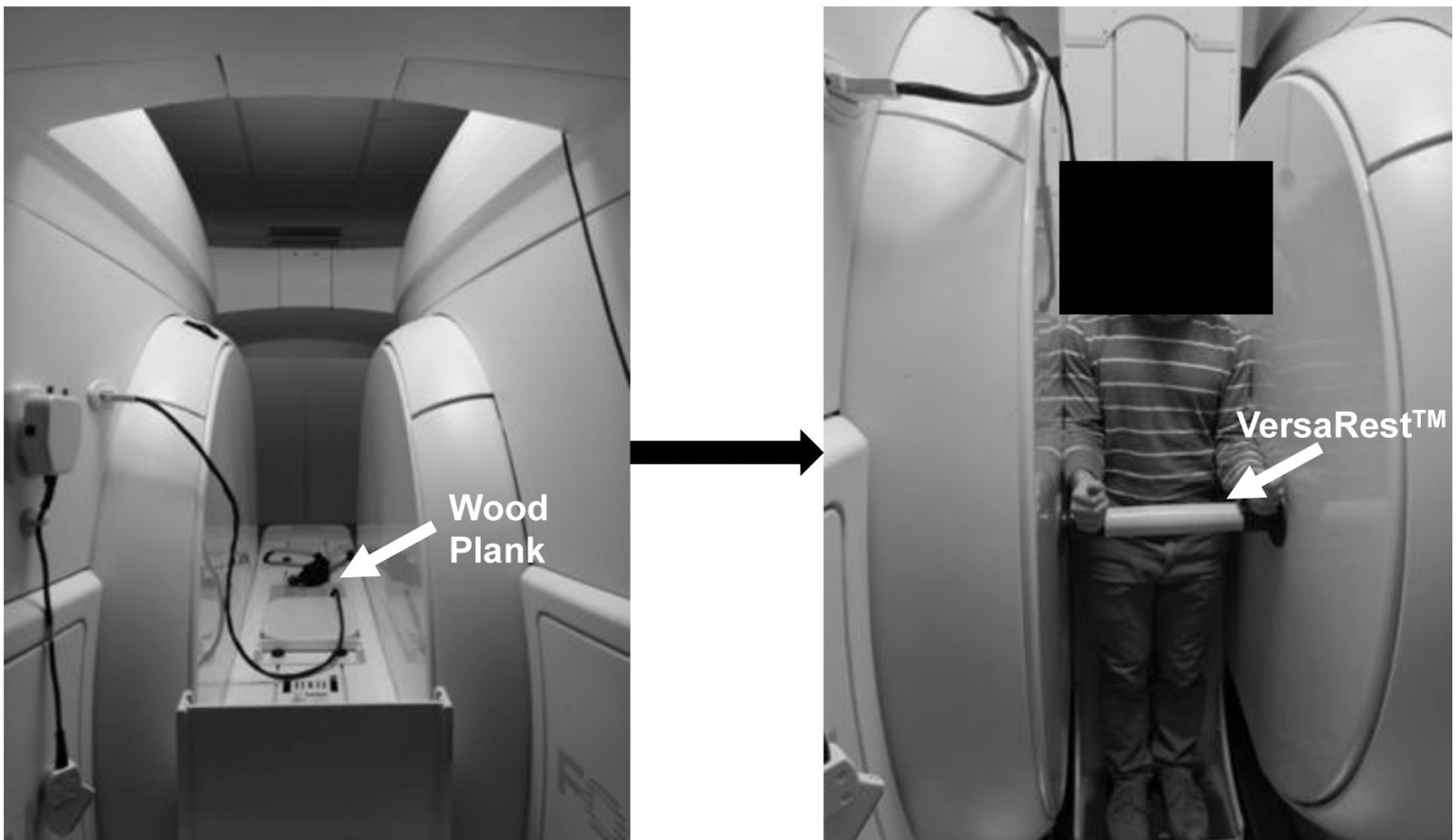

Figure 1:

The pMRI with the table in supine (left) and standing (right) configurations. A wood plank placed behind the participant to provide a continuous flat surface for the lumbar spine and is indicated by the white arrow in the left panel. A VersaRest ${ }^{\mathrm{TM}}$ device to support the arms is indicated by the white arrow in the right panel of the figure. 


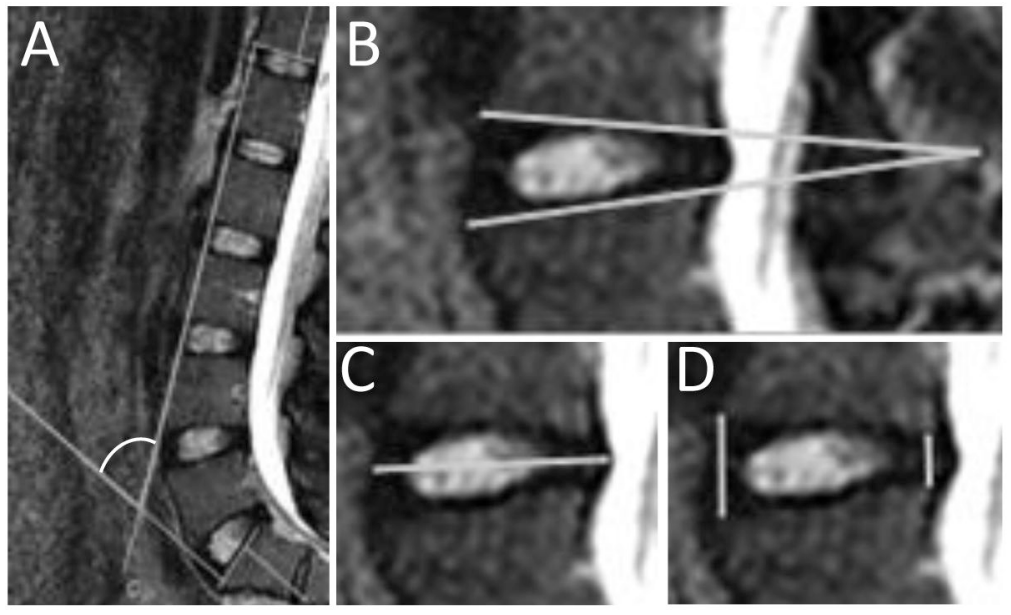

Figure 2:

A representative image of the measurements in this study: A) The four-line Cobb angle, with the white arc indicating the angle of measurement, quantifies the regional spinal alignment. Larger Cobb angles reflect more lumbar lordosis. B) The segmental Cobb angle describes the "wedging" of the IVD. C) The IVD width quantifies the radial bulging that occurs in the IVD. All images are aligned in the same orientation with the left being the anterior side. D) The anterior to posterior height $(\mathrm{A} / \mathrm{P})$ ratio quantifies the relative change of shape of the IVD. 

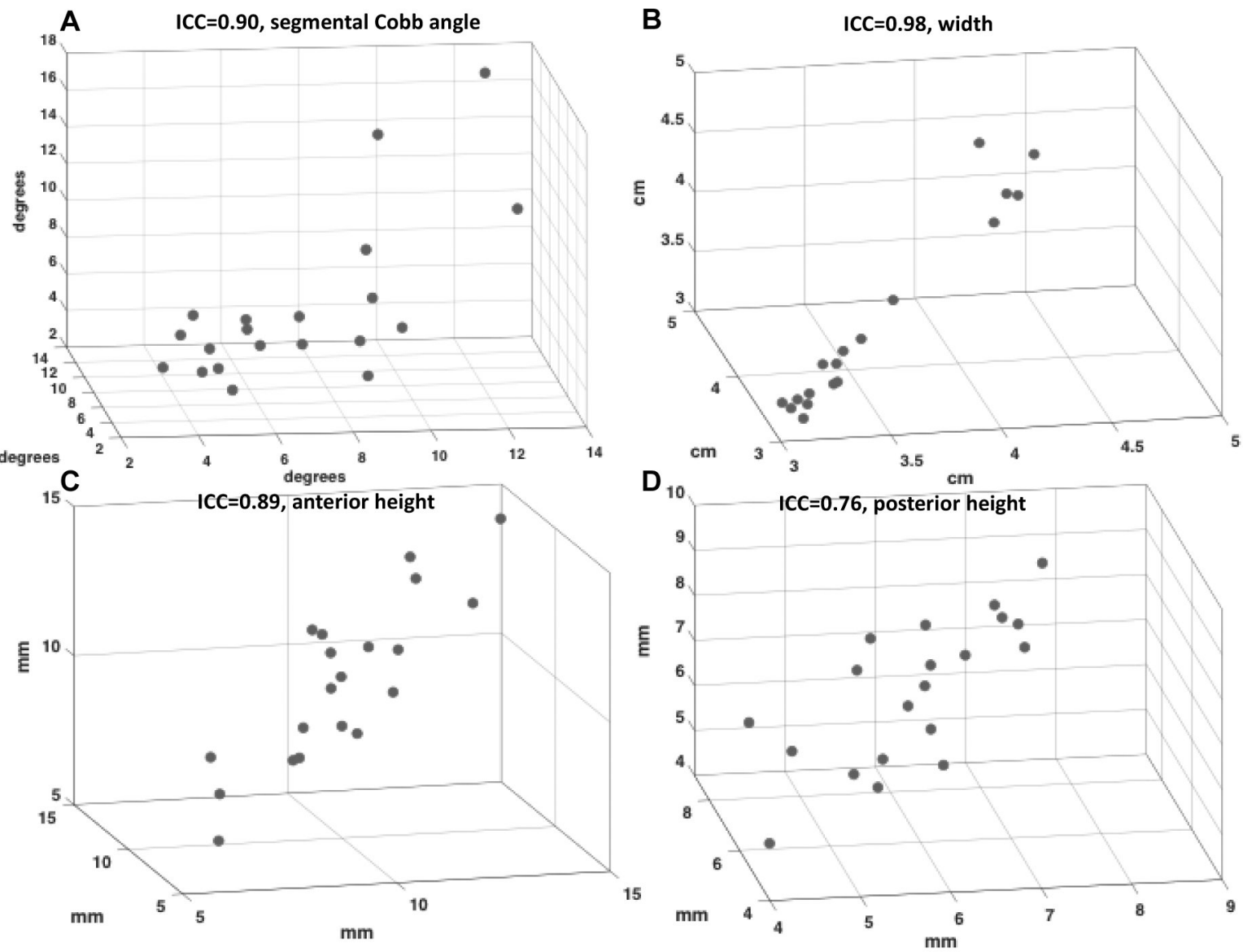

Figure 3:

The intra-observer ICC values are shown for each measurement for individual IVD levels for A) segmental Cobb angle, B) IVD width, C) anterior height, and D) posterior height. Each axis represents an independent observer trial for the measurement. A written rubric for measurement based on anatomical landmarks (segmental Cobb angle, IVD width, the anterior-, and posterior- heights) was used. The intra-observer analysis was performed using measured variables obtained on three different nonconsecutive days. 


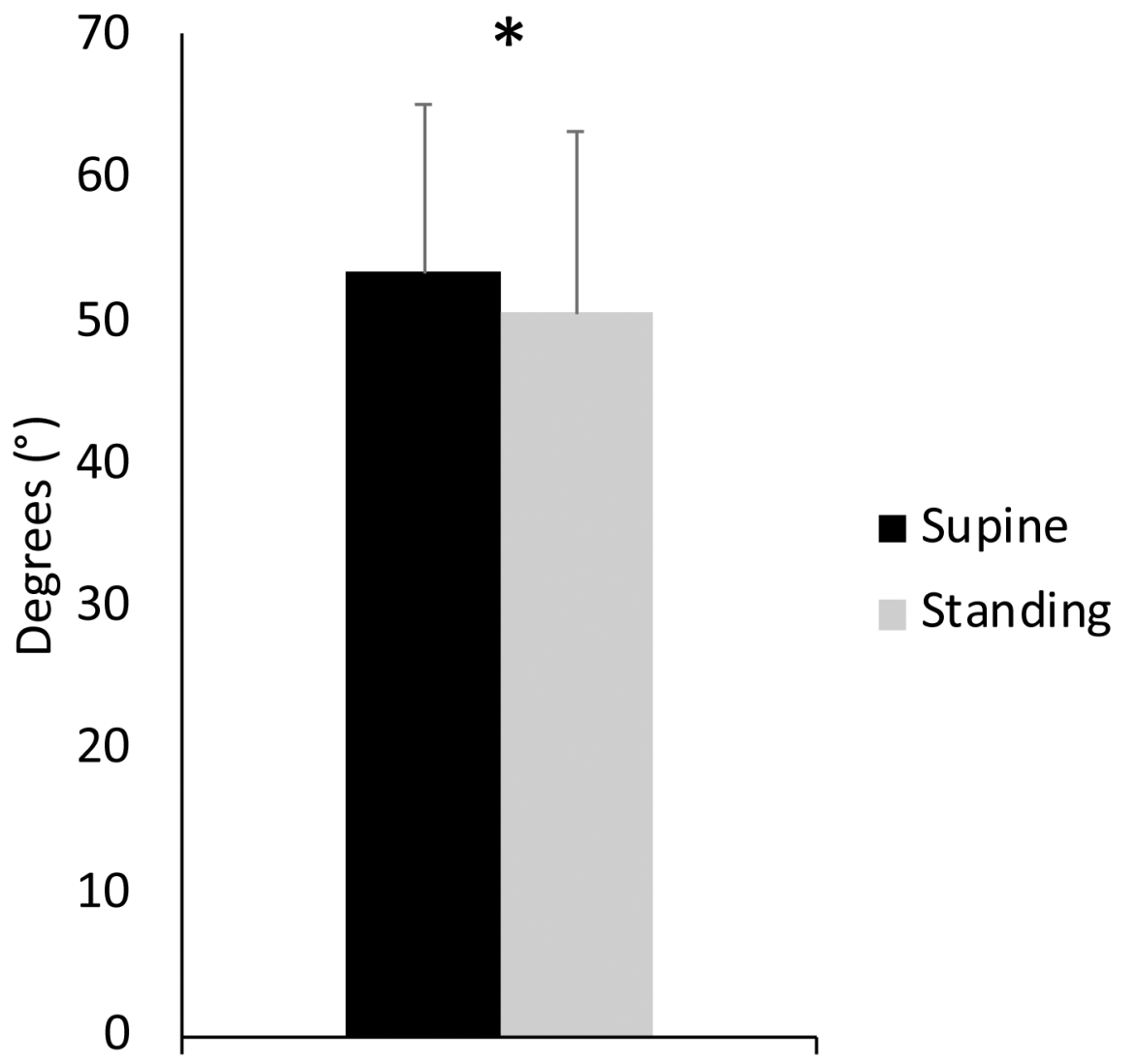

Figure 4:

The Cobb angle for all participants in each position show that lordosis decreases in standing (* : Fisher's LSD test, $P<0.05$ ). Error bars indicate standard deviations. 


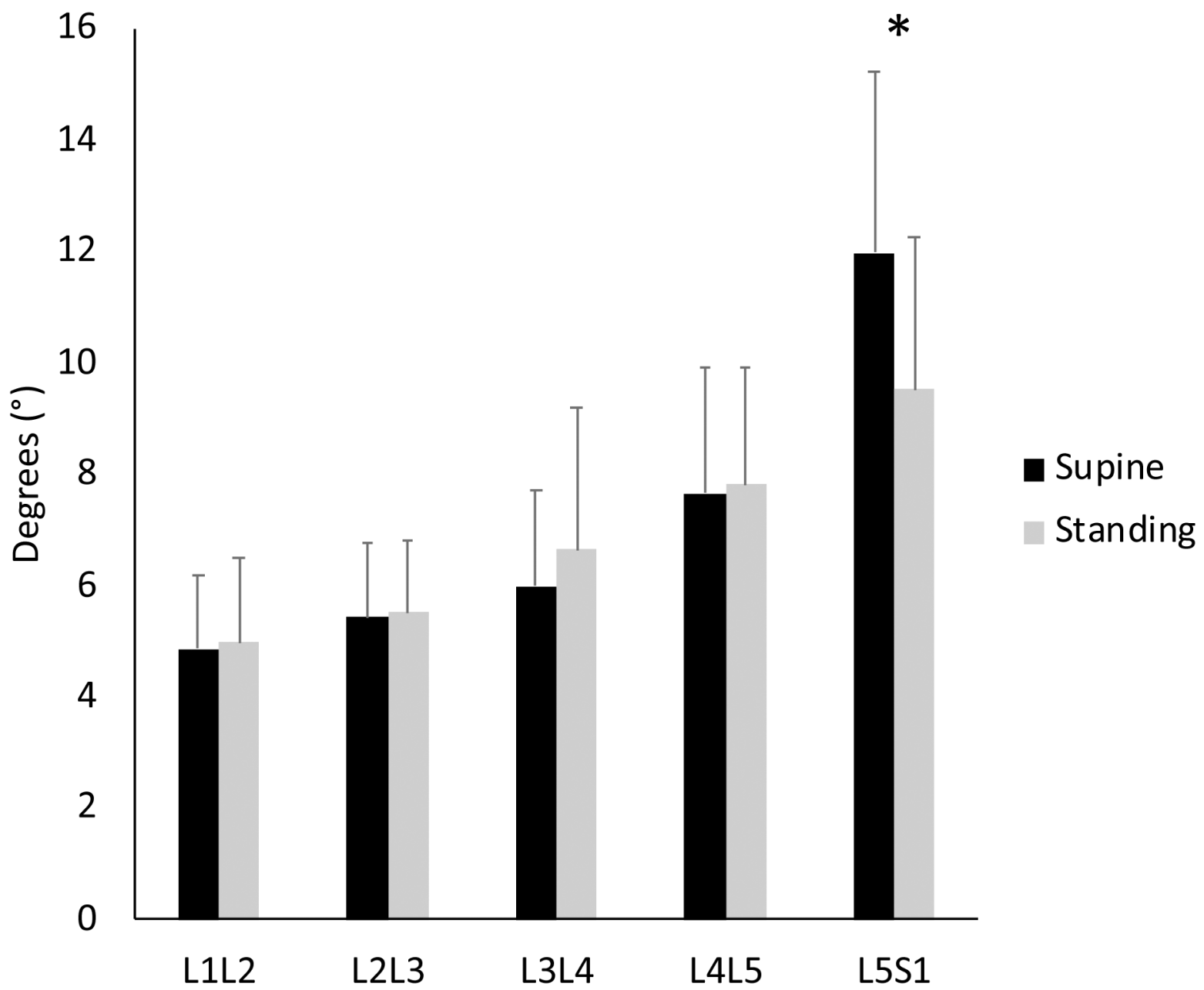

Figure 5:

The mean segmental Cobb angles at each lumbar level for all participants in supine and in standing. The segmental Cobb angle decreased at L5/S1 in standing (* :Fisher's LSD test, $P$ $<0.05)$. Error bars indicate SDs. 


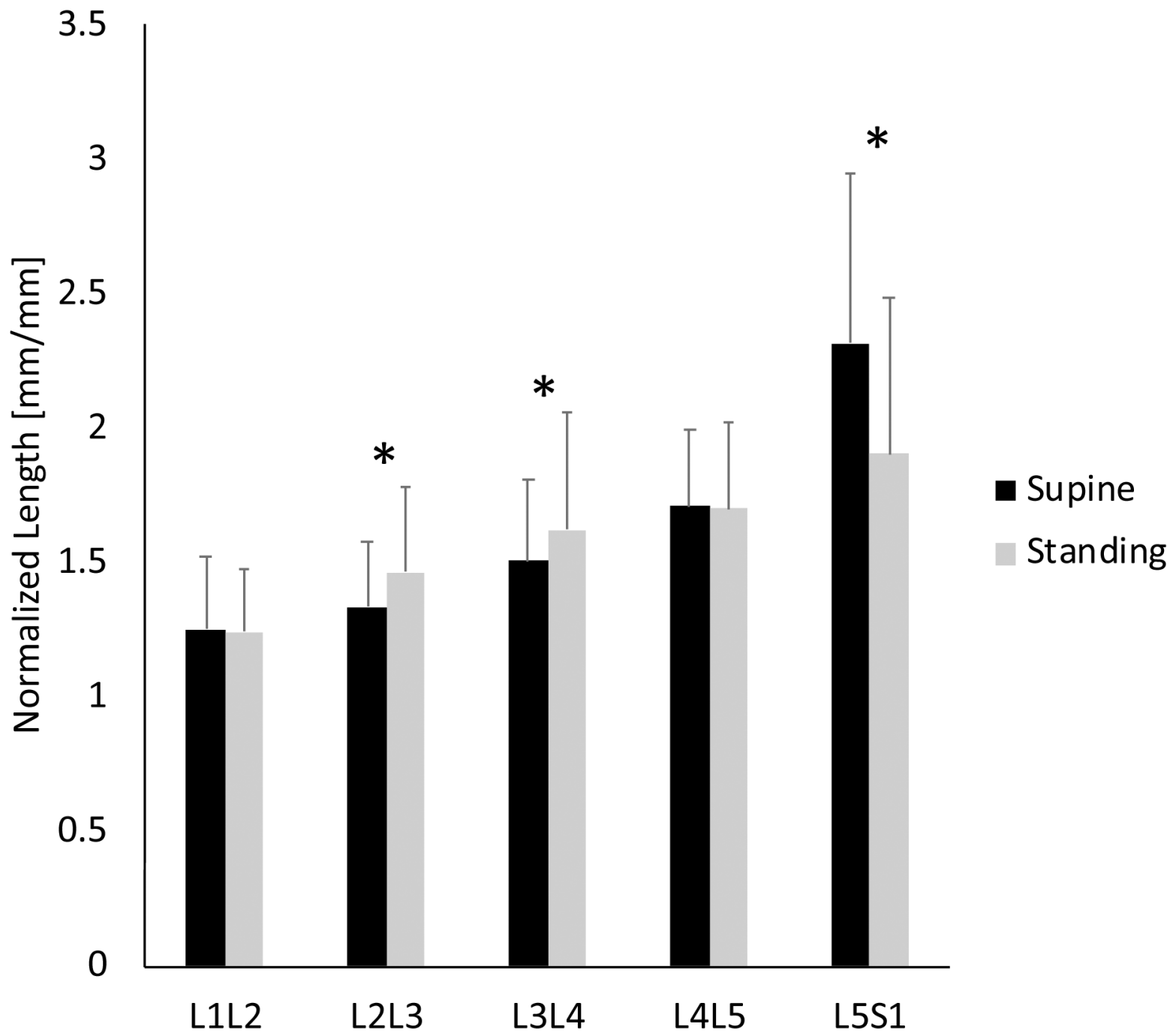

Figure 6:

The A/P ratios show that L2/L3 and L3/L4 increases in standing, and decreased in the L5/S1. (*: Fisher's LSD test, $P<0.05$ ). Error bars indicate SDs. 
Table 1:

Characteristics of the participants presented as the mean (SD). Age $(P=0.81)$ and BMI $(P=0.35)$ are not statistically significant between sexes.

\begin{tabular}{lll}
\hline & Male (19) & Female (21) \\
\hline Age (y) & $24.7(3.5)$ & $24.9(2.1)$ \\
Height $(\mathrm{cm})$ & $179.2(7.4)$ & $164.6(7.8)$ \\
Weight $(\mathrm{kg})$ & $73.3(9.0)$ & $60.4(5.2)$ \\
BMI $\left(\mathrm{kg} / \mathrm{m}^{2}\right)$ & $23.0(2.4)$ & $22.3(1.9)$ \\
\hline
\end{tabular}


Table 2:

$P$-values for the three-way repeated measures ANOVA examining the effects of sex, level, and position and their interactions on the segmental Cobb angle, A/P ratio, and IVD width measurements.

\begin{tabular}{llll}
\hline & & $P$-value & \\
Factors & Segmental Cobb angle & A/P ratio & IVD width \\
Sex & 0.027 & $<0.001$ & $<0.001$ \\
Level & 0.004 & $<0.001$ & $<0.001$ \\
Position & --- & $<0.001$ & $<0.001$ \\
Sex*Level & --- & --- & --- \\
Sex*Position & --- & -- & --- \\
Level*Position & $<0.001$ & $<0.001$ & $<0.001$ \\
Sex*Level*Position & --- & --- & --- \\
\hline
\end{tabular}


Table 3:

The measurements of the regional lumbar spinal alignment and segmental intervertebral disc geometry with significant $P$-values denoted. Values are mean with standard deviations in parentheses.

\begin{tabular}{lllc}
\hline & Supine & Standing & $P$-value \\
\hline Cobbi ingle & $50.6^{\circ}\left(12.6^{\circ}\right)$ & $53.4^{\circ}\left(11.6^{\circ}\right)$ & $<0.05$ \\
L1/L2 Segmental Cobb & $4.86^{\circ}\left(1.3^{\circ}\right)$ & $4.98^{\circ}\left(1.5^{\circ}\right)$ & --- \\
L2/L3 Segmental Cobb & $5.42^{\circ}\left(1.3^{\circ}\right)$ & $5.52^{\circ}\left(1.3^{\circ}\right)$ & --- \\
L3/L4Segmental Cobb & $5.99^{\circ}\left(1.7^{\circ}\right)$ & $6.65^{\circ}\left(2.6^{\circ}\right)$ & --- \\
L4/L5 Segmental Cobb & $7.66^{\circ}\left(2.3^{\circ}\right)$ & $7.81^{\circ}\left(2.1^{\circ}\right)$ & --- \\
L5/S1 Segmental Cobb & $12.0^{\circ}\left(3.2^{\circ}\right)$ & $9.52^{\circ}\left(2.8^{\circ}\right)$ & $<0.05$ \\
L1/L2 A/P ratio & $1.25(0.27)$ & $1.25(0.23)$ & --- \\
L2/L3 A/P ratio & $1.33(0.25)$ & $1.47(0.32)$ & $<0.05$ \\
L3/L4 A/P ratio & $1.51(0.30)$ & $1.62(0.44)$ & $<0.05$ \\
L4/L5 A/P ratio & $1.71(0.28)$ & $1.70(0.32)$ & --- \\
L5/S1 A/P ratio & $2.31(0.63)$ & $1.91(0.58)$ & $<0.05$ \\
L1/L2 IVD width $(\mathrm{cm})$ & $3.33(0.36)$ & $3.36(0.37)$ & --- \\
L2/L3 IVD width $(\mathrm{cm})$ & $3.51(0.42)$ & $3.51(0.39)$ & --- \\
L3/L4 IVD width $(\mathrm{cm})$ & $3.54(0.39)$ & $3.52(0.37)$ & --- \\
L4/L5 IVD width $(\mathrm{cm})$ & $3.60(0.42)$ & $3.56(0.39)$ & --- \\
L5/S1 IVD width $(\mathrm{cm})$ & $3.51(0.43)$ & $3.60(0.43)$ & --- \\
\hline
\end{tabular}

